KOSCHI (King's Outcome Score of Head Injury) was used to assess disability outcomes at discharge from hospital and at long-term follow up. A retinal haemorrhage score (0 to 16) recorded extent of retinal injury. Visual function (normal, visual impairment, no functional vision) was assessed at follow up.

Results Total of 44 children (25 male 19 female) with a median age of 16 weeks. Subdural haemorrhage was seen in 98\% (37\% bilateral); 56/\% had additional injuries on skeletal survey. At discharge, $5 \%$ children had died (KOSCHI 1) or were in vegetative state (KOSCHI 2); 61\% showed features of moderate to severe disability (KOSCHI 3 \& 4); 34\% had a 'good or full recovery' (KOSCHI 5). At long-term follow-up, $14 \%$ showed a worsening of their KOSCHI score. This resulted in overall outcomes of $71 \%$ disability and $29 \%$ full recovery, at a mean age of 5.3 years. Retinal haemorrhages were present in 93\%, 88\% bilaterally (mean score 8). At follow-up, visual impairment was present in 31\%, 7\% of whom had no functional vision.

Conclusion AHT continues to give rise to significant morbidity; this cohort showed a worsening of disability score at long-term follow-up. This indicates disability severity may not be fully appreciated at discharge from hospital, impacting on both provision of accurate prognostic information and in applying targeted follow-up. Visual impairment was more severe in those with a higher retinal haemorrhage score, as might be expected in more extensive injury. As there are few visual outcome studies, this adds to the evidence base, and we would promote that services should ensure visual function is optimised.

\section{G55(P) ANTIPHOSPHOLIPID SYNDROME SHOULD BE CONSIDERED AS A POSSIBLE CAUSATIVE FACTOR IN STROKE IN CHILDREN WITH DOWN'S SYNDROME}

R Bhatt, S Ramdas. Neurology, John Raddiffe Children's Hospital, Oxford, UK

\subsection{6/archdischild-2020-rcpch.41}

Method A three year old girl with Down's syndrome presented to her local DGH with a dense right sided hemiparesis and impaired speech. MRI brain showed an acute left MCA territory stroke with possible multiple events and vascular imaging (MRA) was suggestive of carotid dissection. The time of onset of her symptoms was unclear as she had been unwell with gastroenteritis the previous few days; therefore she was not a suitable candidate for thrombolysis. Investigations done as part of the RCPCH stroke guideline identified positive antibodies to beta2-glycoprotein and cardiolipin raising the possibility of antiphospholipid syndrome. She was initiated on low molecular weight heparin, repeat anticardiolipin and beta2-glycoprotein at three months remained elevated therefore confirming the diagnosis of antiphospholipid syndrome.

Result It is well recognized that patients with Down's syndrome are predisposed to autoimmune conditions, however the association with antiphospholipid syndrome is lesser well known and documented. There are four previous cases reported in literature, but only two children aged 6 years and 11 months at the time of the thrombotic event.

This case report highlights the importance of thinking laterally and considering dual pathology as a possibility in particular when a clear history is not available regarding onset of symptoms. Despite initial investigations clearly providing an aetiology for the stoke i.e. carotid artery dissection in our case, it was imperative to not cease at this point in the diagnostic process. Antiphospholipid syndrome is a lifelong condition requiring long term anticoagulation treatment in order to prevent the debilitating impact of recurrent thromboembolic events such as stroke, DVT and recurrent miscarriages later in life. Therefore, early recognition of this condition in Down's syndrome patients who present with stroke may have the potential to prevent life-long disability.

\section{G56(P) NEUROPSYCHIATRIC PRESENTATIONS OF SYDENHAM'S CHOREA IN THE UK}

${ }^{1} \mathrm{~N}$ Mushet, ${ }^{2} \mathrm{M}$ Morton, ${ }^{2,3} \mathrm{M}$ Punukollu, ${ }^{2} \mathrm{H}$ Minnis, ${ }^{2,4} \mathrm{C}$ Gillberg. ${ }^{1} \mathrm{CAMHS}$ Northumberland, Tyne and Wear NHS FT, Newcastle, UK; ${ }^{2}$ Institute of Health and Wellbeing, University of Glasgow, Glasgow, UK; ${ }^{3}$ CAMHS, NHS Greater Glasgow and Clyde, Glasgow, UK; ${ }^{4}$ Department of Child and Adolescent Psychiatry, University of Gothenburg, Gothenburg, Sweden

\subsection{6/archdischild-2020-rcpch.42}

Sydenham's chorea (SC), a post streptococcal, autoimmune, neuropsychiatric movement disorder, remains the most common cause of chorea worldwide affecting children aged 5-18 years. SC may be the only manifestation of acute rheumatic fever at first presentation, with the implication of potential sequelae, including cardiac complications. SC is rare in UK and a cluster of cases offered an opportunity to examine the course of the disorder.

Aims Patients with a neurologist's diagnosis of SC, referred to Liaison Psychiatry, were interviewed to determine lifetime prevalence of developmental and psychiatric disorders and describe symptoms at onset and relapses of SC and service use.

Methods Subjects, age under 18, identified from psychiatric referral records, were invited with their parent(s) to an interview to establish medical and family history, with construction of a detailed time-line of physical and psychiatric symptoms alongside a child/parent semi-structured, validated, psychiatric diagnostic assessment interview, (KSADS- PL) to explore current and most severe past episode of psychopathology and brief examination with a chorea rating scale.

Results 12/16 (75\%) of referred children, 7 girls, 5 boys, age at interview 10-15 (average 14) attended. Findings highlight pre-existing neuro-developmental vulnerability (6/12), premorbid separation difficulties (4/12). Debilitating symptoms at onset included: wheelchair requirements (6/12), speech impairments (6/12), joint pain (6/12), educational problems, school refusal, severe emotional problems and hallucinations. Choreic movements were not specifically recalled by $7 / 12$. Psychopathology was generally most severe during first episode but 6/12 reported relapse of SC, often with recurrent psychopathology. Over time multiple diagnoses were proposed: separation anxiety (8/12), generalised anxiety (6/12), ADHD (7/12), psychosis (2/12). Some psychopathology remitted but $10 / 12$ had psychiatric symptoms consistent with a diagnosis at interview. Service experience varied: 6/12 were not diagnosed on first presentation. After diagnosis, 8/12 had cardiology and 5/12 rheumatology reviews; physiotherapists saw $8 / 12$, occupational therapists $6 / 12$, speech therapists $3 /$ 12. 11/12 continued on penicillin, $8 / 12$ had received sodium valproate and range of other psychotropic medicines and therapies were offered. 